\title{
Mathematical model of jet control of adaptability of mine turbomachines
}

\author{
Nikolai Makarov \\ Ural State Mining University, 620144 Ekaterinburg, Russia
}

\begin{abstract}
Achieving synergies from high-tech industries in combination with environmental technologies of innovative subsurface use is possible only on the path to the formation of a qualitatively new approach to creation of energy-efficient technologies including assistive technologies. The lack of adaptability of turbomachines providing industrial safety by more than $15 \%$ increases the cost of production of mining and oil and gas complexes of the Russian Federation, reducing their competitiveness due to their low energy efficiency. An equation is derived in the article for calculating the circulation of a circular lattice of aero-gas-dynamic profiles as a function of the energy parameters of the sources and vortex chambers drains associated with the energy efficiency of turbomachines. The dominant influence of jet control over the flow profiles of impeller blades is proved due to the energy characteristics of sources and drains, which are associated with the parameters of the mine network. Improving the methodology for the aerodynamic calculation of circular lattice of aerodynamic profiles and the development of radial aerodynamic schemes with increased adaptability made it possible to derive an absolutely unique vector for the future development of fan construction, fundamentally based on nature-like technologies for energy conversion and transfer. The possibility of a significant increase in aerodynamic loading, adaptability and efficiency of mine turbomachines, made according to radial aerodynamic schemes with vortex chambers built into the blades of the impeller, which perform the functions of adaptive inkjet circulation control devices, is proved. According to the results of the research, the VRVP-8 radial-vortex fan was created, which has $55 \%$ greater energy efficiency in relation to fans of the VME VV type. Currently, turbomachines are being developed based on nature-like technologies in relation to the oil and gas industry.
\end{abstract}

\section{Introduction}

Modern subsurface use strictly requires ensuring industrial and aero-gas-dynamic safety. The aforementioned actualizes the problem of creating mine turbomachines, providing continuously changing parameters of external conditions caused by technological processes, while ensuring a minimum of energy costs [1, 2, 3, 4].

The dynamics of the technological environment parameters reduces the energy efficiency of turbomachines due to their low adaptability, affecting the competitiveness of enterprises. The lack of adaptability of turbomachines actualizes the task of developing a 
methodology for designing and creation of aerodynamically adaptive turbomachines that adequately and at the same time economically reasonably create the necessary fields of air parameters in the technological space, realizing the concept of optimal ecological technology of subsurface use.

\section{Research objective}

The design of turbomachines allows to implement active methods for controlling the circulation of velocity and acceleration of the flow using sources and vortices that form "aero-gas-dynamic profiles" changing the streamlines of the air flow [5-8].

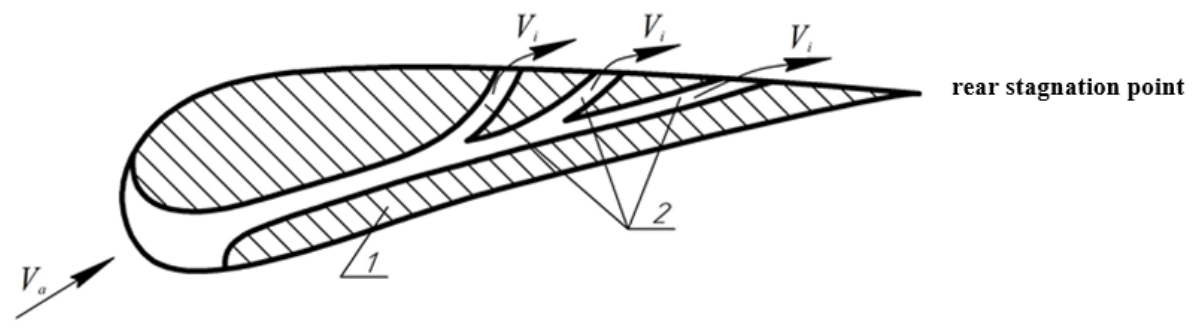

Fig. 1. The aerodynamic profile of the impeller blades of a turbomachine with a flow jet control device.

The jets of the sources 2 slow down or accelerate the air velocity $V_{\mathrm{p}}, V_{\mathrm{T}}$ on the working and back surfaces of the profile of the blade 1 , combining rear stagnation point 3 and the flow separation point 4 . Changing the speed circulation and accelerating the air flow around the profile adapts the aerodynamic parameters of the turbomachine with external conditions. The energy parameters of the Karman vortex track, the position of the flow separation point, and the rear stagnation point in the "aero-gas-dynamic profiles" are determined by the characteristics of the Karman vortex track control devices.

The force interaction of the impeller blades and the air flow in this case occurs through an adaptive aerodynamic system of vortices that ensure the efficiency of the turbomachines and their susceptibility to changes in the external environment, similar to how a bird's wing "interacts" with the air flow during flight. Just as the bird's wing, changing its shape when interacting with an air stream, provides efficient continuous flow around, the system of attached vortices created by vortex chambers of the aero-gas-dynamic profile of a turbomachine, being a mediator in the interaction of the air flow of the interscapular canal with the blades, also provides their continuous flow. Turbomachines designed on the basis of this principle are distinguished by the fact that a wide range of changes in external conditions is nonetheless a nominal effective operating mode for them.

Mixing the jet of the air carried by turbomachine with the blades equipped with the jet control devices for circulation ensures the formation of a stable vortex layer that creates the energy interaction of air and the blade. This scheme provides the susceptibility of the turbomachine to changes in the aerologic situation at the mining enterprise. The energy parameters of the vortex system of aero-gas-dynamic profiles are determined not only by the geometry parameters of the vortex chamber and the blade profiles, but also by the stable feedback of these parameters with the characteristics of the external network, which, in essence, creates an increase in the adaptability of turbomachines, that is, the ability to economically provide the required operating mode. 


\section{Research methods}

The following independent parameters have a dominant effect on the adaptability of mine turbomachines: $\rho$ - air density, $d$ - impeller diameter, $\omega$ - angular velocity, $\Gamma$ - circulation, $\frac{d \Gamma}{d t}$ - acceleration circulation, $\Delta P$ - pressure loss.

In accordance with the theory of similarity, the equation connecting the indicated independent parameters that characterize the aerodynamic process of the formation of the air flow energy interaction of with the aerodynamic profiles of the turbomachine impeller blades and the similarity criterion will be obtained in the form of a dimensionless power dependence $[10,12]$ :

$$
K_{a}=\rho^{\mathrm{a}} \cdot \mu^{\sigma} \cdot \omega^{\mathrm{B}} \cdot\left(\int_{0}^{1} \frac{\partial \Gamma}{\partial S} d S\right)^{\Gamma} \cdot \Gamma^{\text {д }} \cdot \Delta P^{\mathrm{e}}
$$

Using dimension matrices and similarity indicators, we obtain similarity criteria for the aerodynamic process in a turbomachine with adaptive jet control

$$
\begin{gathered}
K_{1}=\frac{\Delta P}{\rho \omega^{2}}=\frac{E_{\text {и }}}{2} \text { - Euler criterion; } \\
K_{2}=\frac{c \int \frac{\partial \Gamma}{\partial S} d S}{\omega d^{2}}=c \int_{0}^{1} \frac{\partial \gamma}{\partial \bar{S}} d \bar{S}=c d \gamma_{\mathrm{T}} \text { - circulation growth indicator; } \\
K_{3}=\frac{\Gamma}{\omega d^{2}}=\pi \gamma \text { - circulation indicator. }
\end{gathered}
$$

From the ratio of the above criteria and similarity indicators, we obtain the aerodynamic adaptability criterion in the following form:

$$
K_{a}=\frac{2 \gamma}{E u} \rightarrow \text { const }
$$

From the formula (3) it can be seen that turbomachines, in which a proportional change in the circulation indicator and Euler criterion is provided, have the greatest adaptability, i.e. $K_{a} \rightarrow$ const

To build a mathematical model of the dependence of the circulation indicator on the characteristics of the jet control device for the flow around the blades, we use the theory of complex numbers and conformal transformations.

The existing theory of blade cascades does not allow to establish the mathematical dependence of the velocity potential around the profiles and energy parameters of the jet circulation control device $[5,6]$.

This article proposes a method for constructing a complex flow potential around a profile due to distributed sources using a hydrodynamic analogy between a jet and distributed sources. Fig. 2 presents a schematic diagram of conformal transformations taking into account the component of the complex potential from distributed sources over the surface of the profile on a circle of unit radius.

In accordance with the generalized problem posed, in a flat situation around a circular lattice with $n_{\text {Л }}$ profiles and $n_{\text {и }}, n_{\mathrm{c}}$ jets of sources and drains of vortex sources on each 
profile, a $\left(n_{\mathrm{n}}+n_{c}+1\right)=(n+1)$-valent streamlined contour is assigned. On the first sheet of a $(n+1)$-valent Riemannian surface - in the physical plane, the studied circular lattice of aero-gas-dynamic profiles is installed, into the vortex chambers of which through the inlet and outlet channels the jets of the air stream created by the rotation of the turbomachine inflow and flow out respectively.

The studies were carried out in a simulated situation in which the circular lattice profiles are rearranged in the form of segments of logarithmic spirals. Since they are streamlines for the flow created by distributed sources in the entire flow region $D_{z}$ on the $(n+1)$-valent Riemannian surface, the flow is stationary and vortex-free, the liquid is ideal, incompressible, weightless and Bernoulli constant does not change.

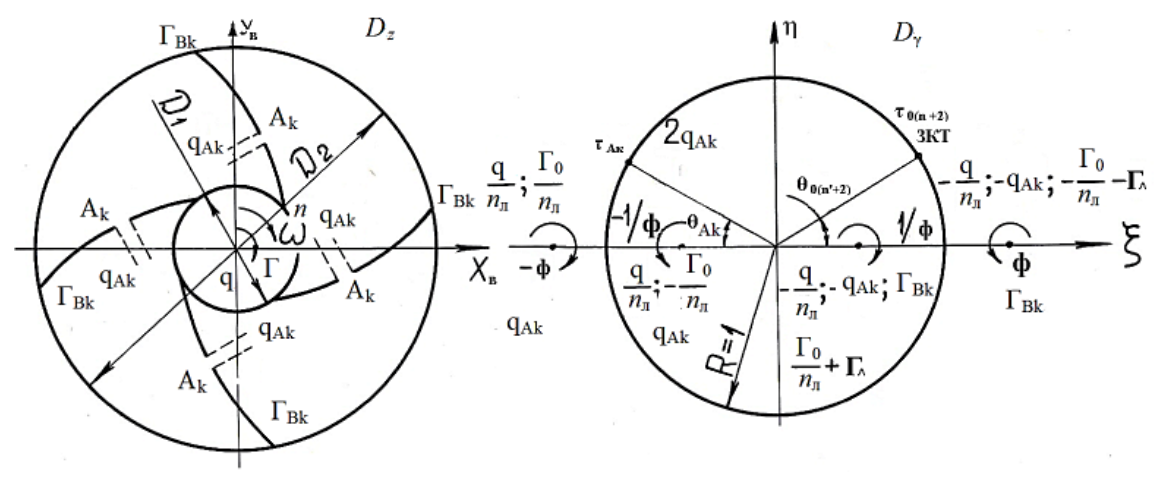

Fig. 2. Layout diagram of conformed transformation sequence $n_{\mathrm{n}}$-valent zone $D_{\gamma}$ В $(n+1)$-valent zone $D_{z}$.

The complex potential associated with the presence of distributed sources on the surface of the profile, taking into account the foregoing and Articles 7, 10, we obtain in the following form

$$
F_{\mathrm{A}_{\kappa}}(\gamma)=\phi_{A_{\kappa}}(\gamma)+i \psi_{A_{\kappa}}(\gamma)=\pi^{-1} q_{A_{K}} \ln \left(\gamma-\tau_{A_{\kappa}}\right)-0,5 \pi^{-1}\left[q_{A_{\kappa}}\left(\ln \left(\gamma^{2}-\Phi\right)+\right) q_{A_{K}} \ln \left(\gamma^{2}-\frac{1}{\Phi^{2}}\right)\right] .
$$

where $q$ - coefficient of the flow rate of the source located in the center of the circular lattice of aerodynamic profiles in the region $D_{z} ; \Gamma_{0}$ - intensity of the vortex (circulation) located in the center of the circular lattice of profiles in the region $D_{z}$, in the presence of preliminary flow swirling at the entrance to the circular lattice; $\Gamma_{\pi}-$ vortex intensity (circulation) around the profile of the circular lattice in the $D_{z}$ plane; $\varphi$ - function of the flow potential in the region $D_{\gamma} ; \Psi$ - stream function (stream line) of the flow in the region $D_{\gamma}$.

The resulting solution for certain $q, \Gamma_{0}, \Gamma_{\mathrm{n}}$, flowrates $q_{A \kappa}$ of sources and drains at points $\tau_{\mathrm{A \kappa}}$ is unique, up to a constant. Accordingly, imagining that there are two solutions: $F_{l}[Z(\gamma)], F_{2}[Z(\gamma)]$ - and using the function $\Delta(\gamma)==F_{I}[Z(\gamma)]-F_{2}[Z(\gamma)]$, is can be easily seen that this function is unique outside the circle and at the circle and at infinity $\operatorname{lm} \Delta(\gamma)=0$. On the basis thereof, by the theorem of uniqueness of solution, the solutions of the DirichletNeumann problem should be $\operatorname{lm} \Delta(\zeta) \equiv 0$, which means that $F_{1}[\mathrm{Z}(\gamma)]-F_{2}[\mathrm{Z}(\gamma)] \equiv$ const.

\section{Results and discussion}

Using the properties of conjugacy, Zhukovsky-Chaplygin-Kutt postulate, results obtained in $[3,9,10]$, additional circulation from the jet control sources is derived by the following 
formula:

$$
\Delta \Gamma_{\mathrm{A}_{\mathrm{\kappa}}}=\sum_{k=1}^{n} q_{\mathrm{A}_{\mathrm{\kappa}}} \frac{\Phi \operatorname{Sin} \theta_{0(n+2)}}{1-\operatorname{Cos}\left(\theta_{\mathrm{A}_{\mathrm{\kappa}}}-\theta_{0(n+2)}\right)} .
$$

Thus, taking into account the formula (3), the condition for ensuring the constancy of the adaptability criterion in the design of turbomachines and experimental studies will be obtained in the following form

$$
K_{a}=\frac{2 \Delta \Gamma_{\mathrm{A}_{\mathrm{K}}}}{E u}
$$

Using [7], the flow coefficients of sources and drains are aero-gas-dynamically interconnected with the pressure in the impeller of the turbomachine associated with the parameters of the external environment.

Based on the presented energy parameters of the distributed sources of circulation jet control, ensuring a constant increase in circulation and the input for vortex formation, contribute to a significant increase in the adaptability parameter of turbomachines, that is, changes in aerodynamic characteristics are adequate to changes in the external environment with maximum functional and economic efficiency.

To confirm the reliability of the adaptability criterion, aerodynamic tests of the lattice of classical and "aero-gas-dynamic profiles" were carried out, taking into account the fact that the ratio of velocity and acceleration circulation around the lattice of profiles and air flow are hydrodynamic analogues of the aerodynamic quality coefficient of the profile and the flow attack angle:

$$
K=\frac{C_{y}}{C_{X}} ; K_{a}=1-\frac{2 \gamma}{E u K}
$$

where $C_{y}, C_{x}, K$ - the coefficient of lifting force, profile resistance and aerodynamic quality of the lattice of the profiles of the impeller blades of a turbomachine [4-6].

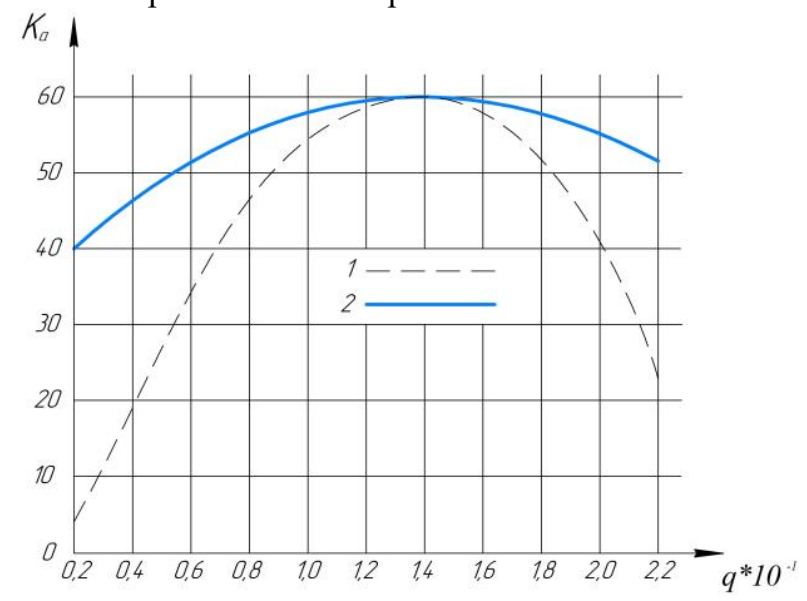

Fig. 3. Dependence of the adaptability criterion of the lattice of the turbomachine blade profiles on the feed: 1 - classic profile, 2 - profile with distributed sources on the profile surface.

The analysis of the test results shown in Fig. 3 shows that distributed sources over the surface of the profile make it possible to increase the adaptability criterion in the sphere of 
economical operation from 45 to $75 \%$, that is, more than $80 \%$.

The increase in the minimum values of the coefficient of aerodynamic quality at the border of the sphere of economical work was 6.1 times from 8 to 49, which corresponds to the increase in the criterion of aerodynamic adaptability in 2.1 times from 0.34 to 0.71 . With equal values of the aerodynamic quality criterion of 0.7 , the increase in the sphere of economical operation will be $83 \%$.

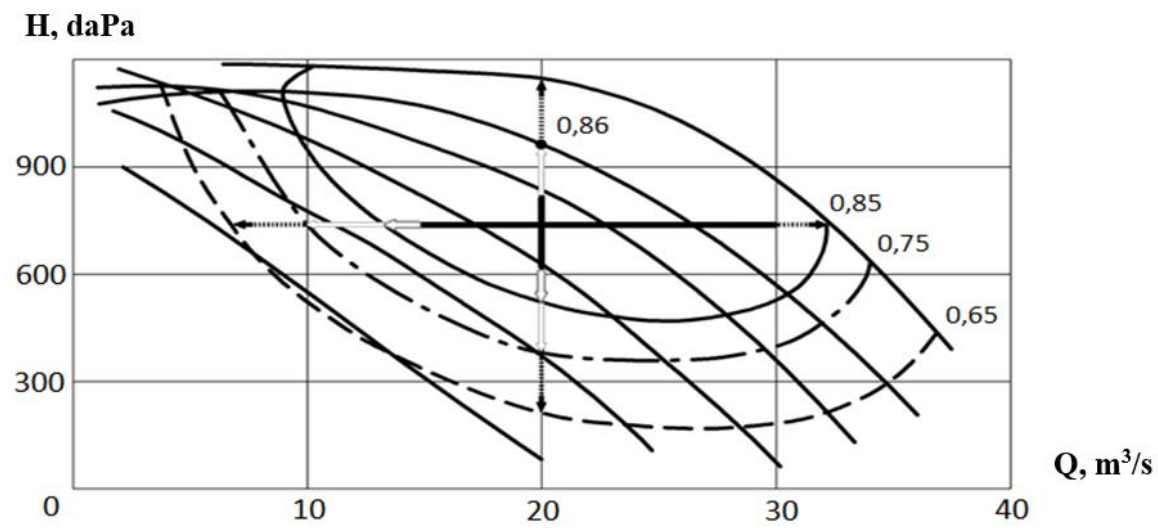

Fig. 4. Aerodynamic characteristic of the VRVP-8 fan with a radial power regulator.

Using the research data, the aerodynamic scheme TS145-25 was obtained, design documentation was developed, on the basis of which the VRVP-8 radial-vortex direct-flow fan was created. Aerodynamic tests of a full-scale sample of the VRVP-8 fan, based on the SMK-Test test center, showed an increase in adaptability by $55 \%$ and a decrease in specific energy consumption by $35 \%$ in comparison with the most advanced domestic mine turbomachines of local ventilation VMEVV-8 (Fig. 4).

\section{Conclusions}

1. Using the hypothesis of the dominant control of the aerodynamic adaptability of turbomachines, a criterion for the adaptability of mine turbomachines was obtained.

2. A mathematical model of the dependence of the adaptability criterion of turbomachines on the circulation indicator and the Euler criterion was developed.

3. Distributed sources on the blade profile can increase the adaptability coefficient of the turbomachine by $80 \%$, which allows a $35 \%$ reduction in the number of fan sizes for covering a mine ventilation network by increasing the area of economic operation.

\section{References}

1. H. L. Wang, G. Xi, J. Y. Li and M. J. Yuan, Proceedings of the Institution of Mechanical Engineers Part A-Journal of Power And Energy, 225, 1143 (2011)

2. V. E. Konovalov, A. I. Semyachkov, V. A. Pochechun, Conference Series: Earth and Environmental Science, 321 (1), 012053 (2019)

3. S. Y. Davydov, N. P. Kosyrev, N. G. Valiev, D. I. Simisinov, V. A. Kurochkin, A. E. Zamuraev, Refractories and Industrial Ceramics, 54 (3), 178 (2013) 
4. N. P. Kosarev, N. V. Makarov, V. N. Makarov, Patent RU 2543638. The way to increase the pressure and efficiency of radial vane turbomachines. Pabl.: 10.03.2015, Bul. №7 (2015)

5. J. P. Gostelow, B. Eng., M.A., Ph.D., M.A.S.M.E., M.R.Ae.S. Professor, Head of School of Mechanical Engineering, The New South Wales Institute of Tehnology, Sydney, Australia, 391

6. S.A.M. Torshizi, A.H. Benisi and M. Durali, ASME Turbo Expo: Turbomachinery Technical Conference and Exposition, Seoul, 1-7 (2016)

7. N. V Makarov, V. N. Makarov, E. E. Franyuk, IOP Conf. Ser.: Earth Environ. Sci. 272 032075 (2019)

8. S.A.M. Torshizi, A. H. Benisi and M. Durali, ScientiaIranica, 24, 707 (2017)

9. V.A. Venikov, The theory of similarity and modeling in relation to the tasks of the electric power industry: textbook for universities, 439 (2014)

10. C.W. Gu, L. Chen, P. Wu and R. Dai, Fluid Machinery, 41, 24 (2013)

11. Y.F. MAO. Numerical Study of Correlation between the Surge of Centrifugal Compressor and the Piping System. Ph.D. Thesis, Xi'an Jiaotong University, Xi'an (2016)

12. M. Zangeneh, N. Amarel, K. Daneshkhah and H. Krain, ASME 2011 Turbo Expo: Turbine Technical Conference and Exposition, Vancouver, British Columbia, Canada, 2167 (2011) 